\title{
Philosophiques
}

\section{Lusignan, Serge, Préface au Speculum Maius de Vincent de Beauvais : réfraction et diffraction, coll. Cahiers d'études médiévales, Montréal-Paris, Bellarmin-Vrin, 1979.}

\section{Claude Gagnon}

Volume 8, numéro 1, avril 1981

URI : https://id.erudit.org/iderudit/203153ar

DOI : https://doi.org/10.7202/203153ar

Aller au sommaire du numéro

Éditeur(s)

Société de philosophie du Québec

ISSN

0316-2923 (imprimé)

1492-1391 (numérique)

Découvrir la revue

Citer cet article

Gagnon, C. (1981). Lusignan, Serge, Préface au Speculum Maius de Vincent de Beauvais : réfraction et diffraction, coll. Cahiers d'études médiévales, Montréal-Paris, Bellarmin-Vrin, 1979. Philosophiques, 8(1), 131-137.

https://doi.org/10.7202/203153ar d'utilisation que vous pouvez consulter en ligne.

https://apropos.erudit.org/fr/usagers/politique-dutilisation/ 


\section{ÉTUDES CRITIQUES}

LUSIGNAN, Serge, Préface au SPECULUM MAJUS de Vincent de Beauvais: réfraction et diffraction, coll. Cahiers d'études médiévales, Montréal-Paris, Bellarmin-Vrin, 1979.

\section{par Claude Gagnon}

Il est rare, même au niveau d'études dites spécialisées, de pouvoir lire un ouvrage dont la portée analytique soit aussi poussée que celle du professeur Lusignan sur la Préface du Speculum Maius. En effet, après un premier chapitre limité à quelques jalons biographiques et à une description sommaire des oeuvres du dominicain, l'auteur consacre tout son travail à l'analyse de la préface que Vincent avait rédigée en tête de son encyclopédie qu'est le Speculum Maius et qu'il avait titrée Libellus Apologeticus. Faisant d'une pierre deux coups, Lusignan nous donne en seconde partie une édition de ce Libellus. Pour employer l'expression utilisée par Lusignan, son livre se veut «une sorte de préface à la préface de la plus grande encyclopédie médiévale» (Introduction, p. 11).

L'auteur remplit bien ses promesses. Après un chapitre ( $\mathrm{Ch}$. II) constitué d'une étude littéraire du Libellus montrant facilement l'étroite dépendance qui a existé entre la rédaction du Libellus et la rédaction de l'encyclopédie, Lusignan se penche successivement sur les deux premiers temps de la double rédaction (Ch. III et IV) pour ensuite démontrer l'interrelation entre le troisième moment de la rédaction du Libellus et la production de la quatrième partie apocryphe du Speculum $(\mathrm{Ch} . \mathrm{V})$. C'est l'ensemble des multiples remarques et découvertes faites au cours de ces analyses qu'il nous retransmet dans son édition du Libellus en éclairant substantiellement notre intelligence du texte rédigé par Vincent et trituré par les continuateurs. Nous allons voir tout de suite que l'entreprise en valait la peine.

Les correctifs d'abord. Concernant la date de parachèvement du Speculum, Lusignan prouve assez facilement que l'hypothèse 
soutenue par les historiens voulant que le «Speculum Maius aurait été à peu près achevé en 1244» (p. 59) doit faire place à une nouvelle supposition fixant «la fin de la rédaction du Speculum autour de 1256-59» (p. 58), et même que «la rédaction de l'ensemble du Speculum Maius se trouvait peu avancée en 1244-46» (p. 70). Lusignan repère tout aussi facilement certains facteurs permettant d'expliquer ce qui apparaissait aux chercheurs comme étant de mystérieux archaïsmes dans l'élaboration du texte du Libellus; par exemple, la transcription par Vincent d'un texte fort antérieur (le ms. latin 13702) dans le chapitre 16 de sa seconde rédaction du Libellus (p. 45). Autre type de rectificatif: Lusignan intervient dans les conclusions d'ordre synthétique d'importants historiens. Il rectifie le jugement de l'historien Échard (XVIII') sur la datation du Speculum morale: contrairement à ce qu'Échard nous avait fait accepter jusqu'ici, plus rien ne prosve qu'il n'existe pas de décalage entre la parution du quatrième Speculum et la troisième version du Libellus remanié pour la circonstance (p. 89), et, autre découverte connexe, la mise en chantier de ces deux faits pas nécessairement reliés ne doit plus être située autour de 1320, comme Échard le pensait, mais bien en plein XIII ${ }^{e}$ siècle (p. 89), peut-être «bien avant 1290» (p. 90), comme l'affirme Lusignan. Dans une perspective plus globale, Lusignan rectifie le jugement de De Rijk (XXed ) qui situait l'ultime reprise du thème victorin de la corrélation des triades (maux, vices, vertus, biens, sciences) dans le commentaire de Raoul de Longchamps de l'Anticladianus, rédigé entre 1212 et 1225. Lusignan nous convainc qu'il faut définitivement prolonger cette histoire de l'idée victorine et verser le Speculum Maius au dossier de cette diffusion (p. 103).

L'apport de Lusignan ne se limite pas à une série de rectifications. Il ajoute aussi au domaine en question des connaissances toutes nouvelles. Il éclaire passablement certains faits dans la chronologie de la vie de Vincent. Ainsi, reprenant les témoignages connus mais les restructurant avec, à la fois, une imagination proprement prospectrice et une prudence toujours scientifique, il solidifie davantage le sens de l'arrivée de Vincent à l'abbaye de Royaumont et, ce faisant, propose avec pertinence une nouvelle hypothèse concernant une contribution des Cisterciens de cette abbaye à son Speculum (p. 71 à 74). 
Tout cela est fait uniquement à partir de l'analyse des différentés rédactions successives du Libellus; analyse dont la minutie est tout simplement exemplaire. Il faut lire comment Lusignan construit ses déductions en se penchant parfois uniquement sur les changements des adverbes dans les phases successives de la rédaction de Vincent dans un même texte, une même phrase (p. 40 pour un glissement de sens concernant le nombre exact de Speculum dans deux rédactions successives du ch. II du Libellus, p. 53 pour évaluer l'antériorité de l'Epistola par rapport au De morali). Toutes ces analyses microscopiques permettant au médiéviste de formuler encore d'autres inductions nouvelles concernant l'aspect technique de l'intelligence du philosophe médiéval. Ainsi l'auteur est en droit d'énoncer dès la fin de son chapitre II, que «Vincent utilisait la technique de juxtaposition des textes, familière à un compilateur, pour la rédaction de ses propres écrits» (p. 49). Toute la démarche est très utile.

Cependant, il y a deux points qui atténuent l'enthousiasme du lecteur. J'ai déjà souligné ailleurs (cf. mon compte rendu de Lusignan dans Science et Esprit, numéro d'avril 1980) la volonté de l'auteur de cet ouvrage de travailler le Speculum en fonction de l'esprit propre à ce dernier. Voilà pourquoi Lusignan place dans le titre même de son étude les termes de «réfraction» et «diffraction» et parle dès la première page de son Introduction dans un vocabulaire qui renchérit sur le thème du Miroir (i.e. Speculum), en disant "notre livre réfléchit cette préface et l'analyse par une décomposition prismatique qui en fait ressortir le sens» (p. 11, les soulignés sont de moi). Mais de "réfraction" et de "diffraction", il n'en est plus jamais explicitement question au-delà de cette première page d'introduction. Qu'est-ce qui est "réfraction» et qu'est-ce qui est «diffraction» dans l'étude? On n'en sait rien, et il nous faut deviner. Si on se fie aux définitions scientifiques admises de ces deux termes, la réfraction concerne la production d'un angle de déviation lorsque la lumière change de milieu, et la diffraction concerne la production de particules lumineuses dans la frange d'ombre lorsque la lumière sort de la périphérie de son champ. Concernant la production du Speculum, Lusignan relate deux phénomènes majeurs. D'abord Vincent de Beauvais a mis en chantier un ouvrage qui s'allongea de façon imprévisible, et ceci de façon démesurée: «au cours de la rédaction, le Speculum suivit 
des tangentes que Vincent n'avait pas prévues. Or il a été établi qu'effectivement le Speculum s'allongea en cours de rédaction» ( $p$. 64); «Vincent ne prévoyait sûrement pas alors la taille que prendrait son encyclopédie dans sa forme finale. (. . .) Le Speculum. (. . .) allait dépasser de beaucoup dans sa forme finale le volume de la Bible» (p. 70). Deuxièmement, le Speculum de Vincent, divisé en trois parties (naturale, doctrinale, historiale), entraîne dans le succès de sa diffusion la production d'un quatrième Speculum apocryphe (morale).

Est-ce à dire que l'allongement imprévisible constitue la réfraction et que la production de l'apocryphe (à l'aide d'ajouts, de modification et multiplication de la numérotation des chapitres du Libellus, de prélèvements de textes et de corrections trop sommaires; p. 78-79) se définit comme la diffraction? Je n'en suis pas du tout certain. Comment alors intégrer cet autre phénomène du Speculum, très justement souligné par Lusignan, et qui constitue le parallèle idéologique entre la disproportion dans le traitement de la nature et de la science au profit de l'histoire dans l'encyclopédie de Richard de Saint-Victor, l'un des maîtres à penser de Vincent, et le sentiment d'insatisfaction qu'exprime ce dernier "face à la trop grande importance que prend la science et la nature dans son Speculum Maius» (p. 106)? Ce dernier phénomène ne relève-t-il pas tout autant de la diffraction dans la mentalité de l'époque et n'est-il pas composé dans sa nature d'une réfraction certaine si on entend par celle-ci une certaine orientation imprévisible dans l'incidence et, par celle-là, la production d'une quantité de lumière dans un champ de savoir jusque-là qualifié par un milieu d'une obscurité quasi totale? L'auteur aurait eu avantage à nous spécifier ce qu'il entendait par les deux substantifs (réfraction et diffraction) qui apparaissent dans le titre de son étude et qui semblent qualifier le miroitement de l'encyclopédie de Vincent sur le savoir de son époque.

Un deuxième point de l'étude de Lusignan laisse le lecteur sur son àppétit. Dans la discussion sur l'origine apocryphe du quatrième Speculum, Lusignan montre le caractère périmé de la recherche d'Échard sur la datation de l'apocryphe en reprenant et compilant les résultats des recherches récentes sur les sources mêmes ayant servi à l'historien du XVIII ${ }^{e}$. Lusignan réussit à nous convaincre qu'Échard s'était trompé sur la datation de 
plusieurs de ces sources, et les nouvelles approximations qu'il nous propose reçoivent en toute logique l'approbation du lecteur. Cependant, si Lusignan démontre qu'il est logique de penser que le quatrième Speculum était en chantier «bien avant 1290" (p. 90), et que «dès 1304 on attribuait quatre Speculum à Vincent, et non plus trois» (p. 86), il explique cette méprise sur l'authenticité du quatrième Speculum, méprise partagée par plusieurs érudits des générations immédiatement postérieures à celle de Vincent, par le fait que «le moyen âge connaissait mal l'encyclopédie de Vincent» (p. 86). C'est ce dernier facteur qui fait problème au lecteur. S'il s'agissait, ici, d'une petite encyclopédie perdue, n'ayant circulé que dans des milieux spécialisés (comme le Speculum Universale de Raoul Ardent), nous comprendrions l'importance de ce facteur de méconnaissance. Mais l'encyclopédie de Vincent connut très tôt et jusque sur le tard une diffusion exceptionnelle. Maurice de Gandillac écrit sur ce point: "le Speculum Maius sera maintes fois recopié et connaîtra six fois les honneurs de la typographie» («Encyclopédies pré-médiévales et médiévales», dans La pensée encyclopédique au Moyen Âge, Neuchâtel, Éditions de la Baconnière, 1966, p. 39). Michel Lemoine, qui rédige le chapitre consacré à l'encyclopédie de Vincent dans ce même volume, est plus explicite: "Ainsi s'explique l'immense audience que connut si longtemps le Speculum majus. (...) un succès dont témoigne l'existence d'environ 80 manuscrits. Cet intérêt survécut largement au Moyen Âge: le Speculum est le premier ouvrage imprimé à Strasbourg. "(Idem., p. 85) Lusignan lui-même parle de «la centaine de codices connus de l'oeuvre» (p. 11). Comment peut-on connaître si mal une oeuvre tant diffusée? Je ne prétends pas que la grande diffusion d'un ouvrage soit garante de sa juste connaissance ou attribution. Trop d'exemples prouvant le contraire existent dans l'histoire de la littérature de cette période. Je suppose seulement qu'une réponse puisse être trouvée pour le cas qui nous intéresse. Et que ce n'est pas parce que "Malheureusement, les Speculum aussi précisément datés sont très rares» (p. 89), qu'il faut passer sous silence, comme Lusignan le fait, la violente contradiction entre la popularité exceptionnelle d'un ouvrage et la possibilité de l'insertion d'un grossier apocryphe à l'intérieur du corpus, et ceci moins de 30 ans après la mort de son auteur. D'autant plus contradictoire que l'apocryphe est nommément grossier, comme nous permet de le penser juste- 
ment la pertinence de l'analyse de Lusignan. C'est ce que nous devons maintenant voir.

Au-delà des rectifications et ajouts apportés par l'auteur, ce dernier, dans les dernières pages de son analyse, accède à une authentique modernité dans ses considérations philosophiques sur la science de Vincent de Beauvais. Il fait toujours plaisir de constater une préoccupation synthétique chez un chercheur versé dans l'analyse de documents anciens. Après avoir critiqué les fondements d'ordre littéraire d'un Échard dans la description que ce dernier fait du caractère falsifié du quatrième Speculum, Lusignan n'affirme pas moins ce caractère, mais en se fondant désormais sur une instance autre que littéraire. Il s'agit de l'instance qu'on nomme aujourd'hui «épistémologique». En effet, l'auteur nous démontre, de façon indéniable, que le Speculum morale ne pouvait pas et n'aurait jamais pu faire partie intégrante du Speculum Maius. Pour la simple mais imperceptible raison aux historiens de la littérature que l'insertion d'un Speculum morale s'oppose doctrinalement au projet du Speculum dans son ensemble. Lusignan a montré la filiation du projet de Vincent par rapport à la nouvelle façon victorine (chez Hugues et chez Richard) de diviser les articulations de la philosophie: "Le Speculum Maius et la première partie du livre de Richard de Saint-Victor sont construits selon une même $W$ eltanschauung dont la terre, la science et l'histoire forment les trois axes fondamentaux.» (P. 106.) Ainsi tout le domaine de l'éthique est déjà contenu dans le Speculum doctrinale (Livres IV à X). Donc, comme Lusignan l'évalue si bien: «La transformation apportée par les auteurs de Morale, bénigne en apparence, détruit en entier l'idéologie qui donne corps au Speculum. C'est pour nous la preuve la plus forte que l'insertion du morale dans le Speculum Maius ne saurait être l'oeuvre de Vincent. Pour répondre à un quelconque besoin d'un miroir moral autonome, on brisa le triptyque habilement mis en place par celui-ci.» (P. 110.)

Il fallait y penser. Malheureusement, longtemps les historiens des sciences n'ont pas tenu compte d'impératifs de ce genre. Ils se contentaient de comparer les possibilités qu'offraient les instances littéraires en néligeant les possibilités épistémiques contenues dans les textes. Lusignan, après avoir compilé et comparé les instances littéraires du Libellus, compare la doctrine 
de ce dernier aux doctrines qui lui ressemblaient et à celles qui ne pouvaient lui ressembler. Il corrige ainsi le jugement de DeRijk, relativise celui du vieux Échard en montrant non seulement le progrès des découvertes littéraires, mais en permettant au philosophe d'avoir son mot à dire dans ce qui concernait d'abord et avant tout la nature et la division de la philosophie. C'est la raison pour laquelle j'aurais bien aimé qu'il se prononce aussi de cette manière sur la contradiction entre l'abondance des sources littéraires et la méprise des érudits concernant l'origine d'un apocryphe d'autant plus grossier qu'il lui fut presque contemporain.

Les victorins et le dominicain innovèrent profondément en rompant avec la division tripartite stoïcienne de la philosophie (physique, logique, éthique) et en proposant une division quadripartite (théorique, pratique, mécanique, logique) (p. 97). Cette révolution dans la classification du savoir et qui permet, comme le fait remarquer justement Lusignan, d'intégrer les arts mécaniques au niveau d'une connaissance au sens propre par le fait qu'ils deviennent «partie» de la philosophie, cette révolution n'est même pas mentionnée dans les deux plus récents ouvrages de synthèse produits par les philosophes des sciences (cf. T. Kuhn, La structure des révolutions scientifiques, Nouvelle bibliothèque scientifique, Paris, Flammarion, 1972; S. Robert, Les révolutions du savoir; théorie générale des ruptures épistémologiques, Science et Théorie, Longueuil, Le Préambule, 1978). Que se passe-t-il dans cette nouvelle discipline de l'épistémologie? Cette science qui est censée se pencher sur son histoire propre et qui oublie encore l'âge moyen de son développement. Je donne la chance au coureur et je fais un procès de bonne intention au professeur Lusignan: c'est peut-être ce scepticisme face à ces synthèses modernes trop englobantes pour être complètes qu'il a voulu exprimer en nous définissant deux opérations épistémologiques (la réfraction et la diffraction), mais en ne nous les repérant pas nommément dans son étude. Afin que nous puissions constater par nous-mêmes comment la question de la science était complexe pour un philosophe des sciences du XIII ${ }^{\mathrm{e}}$. Et jusqu'à quel point le Grand Miroir qu'il s'est efforcé de nous fabriquer, nous l'avons fragmenté en mille morceaux par l'angle d'incidence de notre histoire et de notre oubli.

Département de philosophie Université du Québec à Montréal 